\title{
Tsallis maximum entropy distribution function for stellar rotational velocities in the Pleiades
}

\author{
B.B. Soares, J.C. Carvalho, J.D. do Nascimento Jr., J.R. De Medeiros* \\ Departamento de Física, Universidade Federal do Rio Grande do Norte, 59072-970 Natal, RN., Brazil
}

Received 14 March 2005; received in revised form 2 August 2005

Available online 23 September 2005

\begin{abstract}
In the present study we use a Tsallis maximum entropy distribution law to fit the observed distribution of projected rotational velocity measurements of stars in the Pleiades open cluster. This new distribution function which generalizes the Maxwell-Boltzmann one is derived from the non-extensivity generalization of Boltzmann-Gibbs entropy. We present a comparison between results from the generalized distribution and the Maxwellian law and show that the generalized distribution fits more closely the data.
\end{abstract}

(C) 2005 Elsevier B.V. All rights reserved.

Keywords: Stars; Rotation; Distribution; Main sequence; Open cluster

\section{Introduction}

Rotation is one of the most important observable in stellar astrophysics, driving strongly the evolution of stars, providing also valuable informations on stellar magnetism, mixing of chemical abundances in stellar interior, tidal interaction in close binaries, and engulfing of brown dwarfs and planets. In addition, if the present value of the rotational velocity of stars at a given evolutionary stage reflects the original angular momentum with which they were formed, the behaviour of the distribution of rotational velocity may also be used to study some of the characteristics of the physical processes controlling star formation.

One of the most puzzling questions in stellar astrophysics along the past 50 years is that concerning the nature of the statistical law controlling the distribution of stellar rotational velocity, in spite of the large acceptance that stellar rotation axes have a random orientation [1]. Chandrasekhar and Munch [2] in the middle of the past century were the first to derive analytically the distribution of stellar projected rotational velocity, on the basis of a Gaussian distribution. In their approach, these authors first assumed a parametric form for a function $f(v)$, where $v$ is the true rotational velocity, then computed the corresponding distribution of the projected rotational velocity $V \sin i$ and finally adjusted a set of stellar parameters to reproduce the $V \sin i$ measurements. Two decades later, Deutsch [3] claimed that the distribution of stellar rotational velocities should have the form of a Maxwell-Boltzmann law, by analogy with the rotational velocities of hard

*Corresponding author.

E-mail address: renan@dfte.ufrn.br(J.R. De Medeiros). 
spherical molecules in a box. All these studies followed a conventional view that favours a randomly orientation for stellar rotation axes [1]. Nevertheless, a number of studies have shown a clear discrepancy between theory and observations, where observed distributions are not fitted by a Gaussian or Maxwellian function with a good level of significance. A Gaussian or Maxwellian distribution that fits the fast rotators fails to account for low rotation rates. On the other hand, a fit to slow rotators fails to explain the rapidly rotating stars $[4,5]$.

In fact, construction of unbiased rotational velocity distributions is a hard task. Direct measurements of stellar rotation are based essentially on the rotation period, requiring a huge number of photometric modulation measurements, difficult to achieve for statistically significant sample of stars. Measurements of the projected rotational velocity $V \sin i$ are more straightforward but are, of course, affected by the $\sin i$ projection effect. Nevertheless, if the stellar sample is statistically representative it is then possible to extract an accurate estimate of the distribution of equatorial velocities by normalizing the observed $V \sin i$. Early studies on the nature of the statistics controlling the distribution of stellar rotational velocity were based on $V \sin i$ measurements with poor precision, which, admittedly, can lead to systematic errors on the final analyses for low $V \sin i$ values. In addition, most of the early works have not taken into consideration the different physical mechanism that may affect rotation along the stellar evolution.

The molecular clouds where stars are formed are very irregular, with turbulent motions, leading to the assumption of chaotic star formation processes, including collapse and fragmentation of large gas clouds and turbulence in smaller ones. A random orientation of rotation axes is expected on the basis of these stellar formation processes. A chief argument to support the hypothesis that $i$, the angle of inclination between axis of rotation and the line of sight, is distributed randomly is the fact that rotational velocities of stars seems to be independent of their galactic coordinates. Nevertheless, stars do not interact in the same way as hard spherical molecules in a box, with a large number of physical mechanisms affecting the rotation once stars are formed. Among these mechanisms one can underline: angular momentum transfer either from the star to the surrounding accretion disk [6] or to a stellar wind originating at the boundary between the disk and stellar magnetosphere [7], tidal interaction and mass exchange in close binary systems [8], engulfing of planets or brown dwarfs [9], changes in the moment of inertia as a result of changes in stellar radius and external structure and possible decoupling within the star between rotation of its external shells and its core. Further, different studies have pointed for a dependence of rotation of specific group of stars on their position in the Galaxy $[10,11]$.

In the present study, we show that the question of the nature of the distribution of stellar rotational velocity, at least for low-mass stars in the Pleiades open cluster, is not simply a question of which mathematical function model is used, but it depends primarily on the statistical mechanics applied, which should be general enough to take into account the changes in rotation with time. For this purpose, we have used the Tsallis maximum entropy distribution law to fit the observed rotational velocity distribution of the stars in the Pleiades. This new distribution function (hereafter $q$-Maxwellian) generalizes the Maxwell-Boltzmann function [12], and arises from the non-extensive statistical framework first proposed by Tsallis [13] and applied successfully to different astrophysical problems, like matter distribution of self-gravitating system [14-16], stellar polytropes [17], Lévy- and correlated-like anomalous diffusions [18], solar neutrino fluxes [19], cosmological model [14], dynamic linear response theory [15], velocity distribution of galaxy clusters [16], gravothermal catastrophe [17] and Jeans gravitational instability [20]. According to Tsallis et al. [21], within the generalized thermostatistics, the ubiquity and robustness of Lévy distributions in nature follow naturally from generalized central limit theorem. Normal, Gaussian-type, diffusion and anomalous, Lévy-type, superdiffusion now can be unified in a simple picture. The reader is also referred to Tsallis [22] and Abe and Okamoto [23] for a more comprehensive discussion on Tsallis non-extensive statistics and its applications.

One should note that we have focused our first attempt on the Pleiades stars, since stellar clusters are important laboratories for the study of stellar parameters because their stars show some similar properties as age and metallicity, while others as rotational velocity may differ from star to star. This well-studied, relatively young stellar cluster (its age amounts to 100 million years) is about $13 \mathrm{pc}$ across, encompassing $\sim 1000 M_{\odot}$ in stars. We firstly give a brief account of the $q$-Maxwellian function, then we determine the best fit for the observed distribution of the projected rotational velocity $V \sin i$ in different mass ranges of the Pleiades stars. Finally, we give some conclusions and perspective of future work to further clarify the problem. 


\section{2. q-Maxwellian distribution function}

The principle that guided Maxwell arguments in establishing his celebrated Gaussian distribution of velocities is not a privilege of the exponential function, but it is shared by an entire family of power-law functions. These are the $q$-exponentials $\left.\exp _{q}(f) \equiv[1+(1-q) f)\right]^{1 /(1-q)}$ where $f$ is a function of random variables, which includes the standard exponential as the limiting case when $q=1$. This provides a remarkable bridge with non-extensive statistical mechanics, where the $q$-exponentials play a fundamental role (just as the exponential does within Boltzmann-Gibbs statistical mechanics). This observation enabled the establishment of the $q$-generalized classical energy equipartition theorem [24] yielding also a power law type distribution in which the variable parameter is the kinetic energy. On the other hand, Latora et al. [25] have used the rotational velocity as the variable parameter in his work on the dynamics of a Hamiltonian system of $N$ planar classical spins, whereas Campa et al. [26] have used the same framework in the study of rotators interacting through an infinite range potential.

In what follows we illustrate the application of the $q$-Maxwellian distribution function in the context of the present study.

Deutsch [3] has considered the distribution function for the magnitude of a vector that has random orientation. For this, it is required to find the distribution function of a positive scalar $\omega$, which is the magnitude of a vector $\vec{\omega}$. We assume that the distribution of $\vec{\omega}$ is isotropic. We also assume that if it is decomposed into components along Cartesian axes, the distribution of any component is independent of the other components.

Deutsch has defined $\Omega$ as the non-dimensional quantity $j \omega$, where $j$ is a parameter with the dimension of $\omega^{-1}$, so

$$
\vec{\Omega}=\Omega_{x} \hat{i}+\Omega_{y} \hat{j}+\Omega_{z} \hat{k} .
$$

The probability that $\Omega_{x}$ lies in the interval $\left[\Omega_{x}, \Omega_{x}+\mathrm{d} \Omega_{x}\right], \Omega_{y}$ in $\left[\Omega_{y}, \Omega_{y}+\mathrm{d} \Omega_{y}\right]$ and $\Omega_{z}$ in $\left[\Omega_{z}, \Omega_{z}+\mathrm{d} \Omega_{z}\right]$ is then

$$
F(\Omega) \mathrm{d}^{3} \Omega=f\left(\Omega_{x}\right) f\left(\Omega_{y}\right) f\left(\Omega_{z}\right) \mathrm{d} \Omega_{x} \mathrm{~d} \Omega_{y} \mathrm{~d} \Omega_{z},
$$

with $\Omega=\sqrt{\Omega_{x}^{2}+\Omega_{y}^{2}+\Omega_{z}^{2}}$. It is straightforward [3] to show that $F(\Omega)$ is the standard Maxwellian distribution

$$
F(\Omega)=\frac{4}{\sqrt{\pi}} \Omega^{2} \exp \left(-\Omega^{2}\right) .
$$

Nevertheless, we can analyse the problem within the non-extensive framework proposed by Tsallis [13], modifying the basic hypothesis of statistical independence between the distributions associated with the components of $\vec{\Omega}$. As pointed out by Silva et al. [12], the independence between the three velocity components do not hold in systems with long-range interaction, where the non-extensive character is observed. Taking into account such arguments, Silva et al. [12] have proposed the following generalization for Eq. (1),

$$
F(\Omega) \mathrm{d}^{3} \Omega=\exp _{q}\left(\ln _{q} f\left(\Omega_{x}\right)+\ln _{q} f\left(\Omega_{y}\right)+\ln _{q} f\left(\Omega_{z}\right)\right) \mathrm{d} \Omega_{x} \mathrm{~d} \Omega_{y} \mathrm{~d} \Omega_{z},
$$

where the $q$-exponential $\exp _{q}(f)$ and $q$-logarithm $\ln _{q}(f)$ functions are defined as

$$
\exp _{q}(f)=[1+(1-q) f]^{1 /(1-q)} \text { and } \ln _{q}(f)=\frac{f^{1-q}-1}{1-q}
$$

Note that in the limit $q=1$, $\exp _{q}(f)$ and $\ln _{q}(f)$ reproduce the usual exponential and logarithm functions recovering the standard form of (1).

The partial differentiation of the $q$-log of (3) with respect to $\Omega_{i}$ yields

$$
\frac{\partial \ln _{q} F}{\partial \Omega_{i}}=\frac{\partial}{\partial \Omega_{i}}\left(\ln _{q} f_{x}+\ln _{q} f_{y}+\ln _{q} f_{z}\right)
$$


since $\exp _{q}\left(\ln _{q}(f)\right)=\ln _{q}\left(\exp _{q}(f)\right)=f$, where $i=x, y, z$. Equivalently,

$$
\frac{\Omega_{i}}{\chi} \frac{F^{\prime}(\chi)}{F^{q}(\chi)}=\frac{\partial}{\partial \Omega_{i}}\left(\ln _{q} f_{i}\right)
$$

where $\chi=\sqrt{\Omega_{x}^{2}+\Omega_{y}^{2}+\Omega_{z}^{2}}$ and $F^{\prime}(\chi)$ is the total derivative of $F(\chi)$.

Defining $\Phi(\chi) \equiv(1 / \chi) F^{\prime}(\chi) / F^{q}(\chi)$, we can rewrite (5) as

$$
\Phi(\chi)=\frac{1}{\Omega_{x}} \frac{\partial}{\partial \Omega_{x}}\left(\ln _{q} f_{x}\right)=\frac{1}{\Omega_{y}} \frac{\partial}{\partial \Omega_{y}}\left(\ln _{q} f_{y}\right)=\frac{1}{\Omega_{z}} \frac{\partial}{\partial \Omega_{z}}\left(\ln _{q} f_{z}\right) .
$$

This equation can be satisfied only if all terms are equal to a constant that does not contain the $\Omega$-components. Thus, we can make $\Phi(\chi)=-\gamma$, that is,

$$
\frac{1}{\Omega_{i}} \frac{\partial}{\partial \Omega_{i}}\left(\ln _{q} f_{i}\right)=-\gamma .
$$

Hence, the solutions of Eq. (7) for $f\left(\Omega_{i}\right)$ are given by

$$
\ln _{q} f_{i}=-\frac{\gamma \Omega_{i}^{2}}{2}+\ln _{q} A
$$

where $A$ is a constant of integration. Taking the $q$-exponential we obtain

$$
f\left(\Omega_{i}\right)=\left[1+(1-q)\left(\ln _{q} A-\frac{\gamma \Omega_{i}^{2}}{2}\right)\right]^{1 /(1-q)} .
$$

Defining a new constant as

$$
\frac{2}{\sigma^{2}} \equiv \frac{\gamma}{1+(1-q) \ln _{q} A}=\frac{\gamma}{A^{1-q}},
$$

where the parameter $\sigma$ is the width of the $q$-Maxwellian, and substituting it in (9), results

$$
f\left(\Omega_{i}\right)=A_{q}\left[1-(1-q) \frac{\Omega_{i}^{2}}{\sigma^{2}}\right]^{1 /(1-q)} .
$$

Here, we have introduced the sub-index $q$ to make explicit the $q$-dependence of $A$. Eq. (10) recovers the standard exponential term of the Gaussian function for $q=1$.

Then the probability of finding $\Omega$ in the interval $[\Omega, \Omega+\mathrm{d} \Omega]$ can be determined as follows:

$$
F(\Omega)=\int f(\Omega) \mathrm{d}^{3} \Omega .
$$

Putting $\mathrm{d}^{3} \Omega=\Omega^{2} \sin \theta \mathrm{d} \theta \mathrm{d} \varphi \mathrm{d} \Omega$, for a given $\Omega$ we obtain

$$
F_{q}(\Omega)=\iint A_{q} \Omega^{2}\left[1-(1-q) \frac{\Omega^{2}}{\sigma^{2}}\right]^{1 /(1-q)} \sin \theta \mathrm{d} \theta \mathrm{d} \varphi,
$$

which after integrating gives

$$
F_{q}(\Omega)=4 \pi A_{q} \Omega^{2}\left[1-(1-q) \frac{\Omega^{2}}{\sigma^{2}}\right]^{1 /(1-q)} .
$$

Since the standard distribution of the true rotational velocity $V$ for a star sample is $F(V) \propto V^{2} \mathrm{e}^{-V^{2}}$, as shown by Deutsch [3], the standard observed distribution of the projected rotational velocity $V \sin i$, for a random orientation of axes, must be given by $\phi(y) \propto y \mathrm{e}^{-y^{2}}$ [27], with $y \equiv V \sin i$. Henceforth, the $q$-distribution $\phi_{q}(y)$ should reproduce the standard one, in the same way as $F_{q}(V)$ recovers $F(V)$ in the $q=1$ limiting case. 
Therefore, we propose the following distribution function for the observed stellar rotational velocities:

$$
\phi_{q}(y)=B_{q} y\left[1-(1-q) \frac{y^{2}}{\sigma^{2}}\right]^{1 /(1-q)},
$$

where $B_{q}$ is a $q$-dependent constant which should be determined analytically from the normalization of $\phi_{q}(y)$. One can note that for values of $q$ less than unity, the positiveness of the distribution function implies a natural cut-off in the maximal allowed value for $y$. This limit is showed in Eq. (13).

\section{The data set}

The rotational velocities $V \sin i$ used in the present analyses were taken from the rotational survey for the Pleiades stars carried out by Queloz et al. [28]. Our sample amounts to 219 stars of the original sample given by those authors, from where we have excluded stars with only an upper limit in $V \sin i$. All the selected objects are low-mass stars and provide a complete and unbiased rotation data set for stars in the $B-V$ range (0.4-1.4) corresponding to a mass range from $0.6 M_{\odot}$ to $1.2 M_{\odot}$. For a complete discussion on the observational procedure, calibration and error analysis the reader is referred to Queloz et al. [28]. Nevertheless, let us underline that individual errors in $V \sin i$ measurements are better than about $1 \mathrm{~km} / \mathrm{s}$ and do not play a significant role on the observed distributions as shown in Fig. 1.

\section{Results}

For the present statistical study, we have made three different analysis. In the first one (A) we take all stars in the whole $B-V$ interval of the sample (0.4-1.4). In the second (B) we divided the sample in two sub-samples of $B-V$, namely $(0.40-0.90$ and $0.90-1.40)$ while in the third (C) we took three sub-samples corresponding to $B-V$ intervals $(0.40-0.73,0.73-1.07$ and $1.07-1.40)$.

We have normalized the $V \sin i$ values with respect to the average in each sub-sample. In addition, in order to avoid biases in constructing of the frequency histograms due to arbitrary choices of bin range, we have

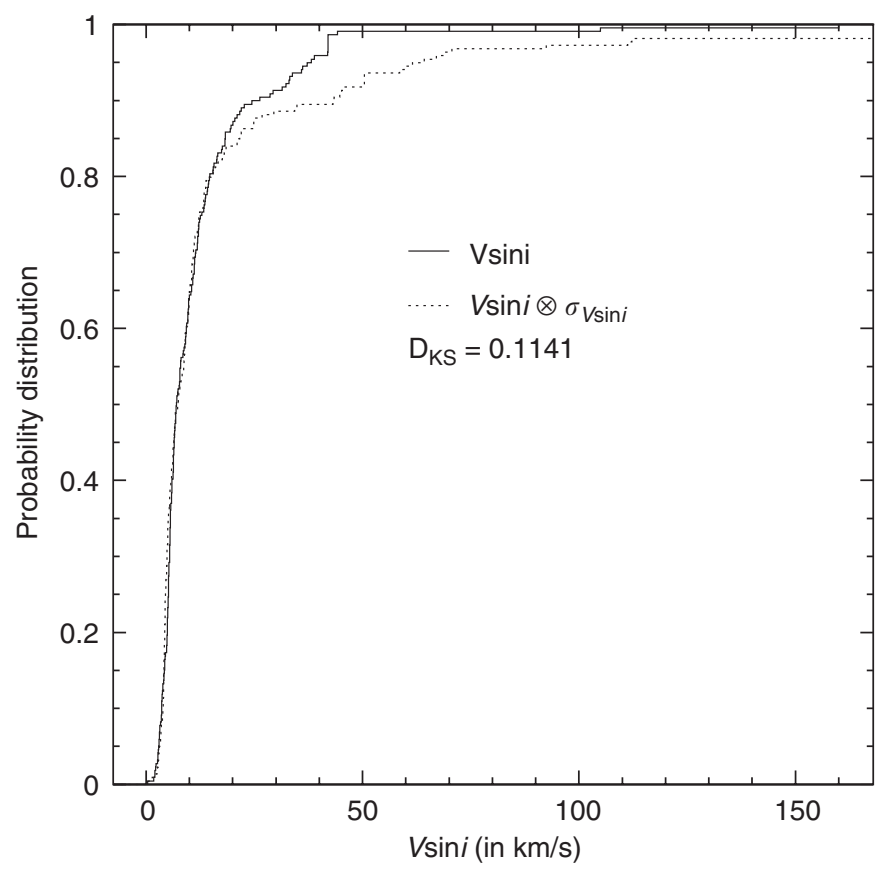

Fig. 1. Comparison between $F(V \sin i)$ probability distribution and its convolution with measurement errors for the star sample. The Kolmogorov-Smirnov statistics $D_{K S}$ is the largest distance between the two distributions. 
decided to study the observed cumulative distribution of the rotational velocities, $F(V \sin i)$, and compare it with the probability distribution function in (12), namely

$$
\Phi_{q}(y)=\frac{\int_{0}^{y} y\left[1-(1-q) \frac{y^{2}}{\sigma^{2}}\right]^{1 /(1-q)} \mathrm{d} y}{\int_{0}^{y_{\max }} y\left[1-(1-q) \frac{y^{2}}{\sigma^{2}}\right]^{1 /(1-q)} \mathrm{d} y},
$$

where

$$
y_{\max }=\left\{\begin{array}{ll}
\frac{\sigma}{\sqrt{1-q}} & \text { if } q<1 \\
\infty & \text { if } q \geqslant 1
\end{array}\right\} .
$$

This distribution function was used to fit the observational data, to obtain the best $\Phi_{q}(y)$ and, consequently, the best $q$-value and $\sigma$-value for each observed cumulative distribution using the Kolmogorov-Smirnov statistical test.

The values of $q$ are shown in Fig. 2 as a function of the average value of $B-V$. The first thing to notice is that, in all three cases (A, B and $\mathrm{C}$ ), the $V \sin i$ distributions of the Pleiades stars effectively do not obey a standard Maxwellian function. For instance, in the upper panel (entire sample) the observed rotation
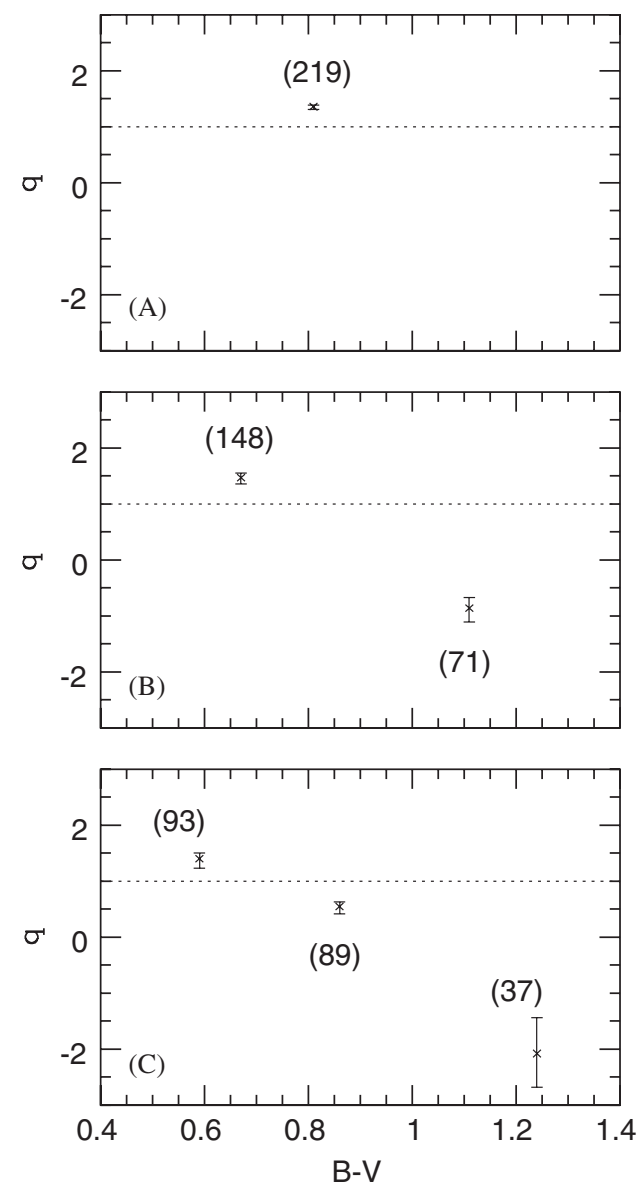

Fig. 2. Best $q$-values as a function of average value of $B-V$ in each interval for different divisions of the star sample. In each panel, we show the number of stars for each $B-V$ interval, the $q=1$ line representing the standard Maxwellian function, and the error bars corresponding to 0.05 significance level for the best $\sigma$ value determined. 
Table 1

Best $q$-values determined by using Kolmogorov-Smirnov test for each $B-V$ interval corresponding to the panels in Fig. 2

\begin{tabular}{|c|c|c|c|c|c|}
\hline Panel & $\begin{array}{l}\Delta(B-V) \\
\langle B-V\rangle\end{array}$ & $q$ & $\sigma$ & $P_{\max }$ & $N$ \\
\hline A & $\begin{array}{l}0.40-1.40 \\
0.81\end{array}$ & 1.36 & 0.57 & 0.21 & 219 \\
\hline \multirow[t]{2}{*}{ B } & $\begin{array}{l}0.40-0.90 \\
0.67\end{array}$ & 1.47 & 0.52 & 0.86 & 148 \\
\hline & $\begin{array}{l}0.90-1.40 \\
1.11\end{array}$ & -0.86 & 1.70 & 0.18 & 71 \\
\hline \multirow[t]{3}{*}{$\mathrm{C}$} & $\begin{array}{l}0.40-0.73 \\
0.59\end{array}$ & 1.40 & 0.63 & 0.96 & 93 \\
\hline & $\begin{array}{l}0.73-1.07 \\
0.86\end{array}$ & 0.55 & 1.27 & 0.20 & 89 \\
\hline & $\begin{array}{l}1.07-1.40 \\
1.24\end{array}$ & -2.08 & 1.79 & 0.47 & 37 \\
\hline
\end{tabular}

distribution is fitted by a generalized distribution function with $q=1.36_{-0.05}^{+0.03}$ and $\sigma=0.57$. Moreover, when we examine the sub-samples (cases $\mathrm{B}$ and $\mathrm{C}$ ), we observe that $q$ does not stay constant, but is a decreasing function of $B-V$. In general, stars with $B-V>0.80$ tend to have a $V \sin i$ distribution best fitted by a $q$ Maxwellian with $q<1$ and, for higher values of $B-V, q$ becomes negative.

A detailed summary of our statistical analysis is shown in Table 1. There, for each case, we present the best results determined for $q$ and $\sigma$, the $B-V$ interval, $\langle B-V\rangle$, the maximum probability for the pair $(q ; \sigma)$ and the number of stars in each analyzed $B-V$ interval.

It is instructive to compare the distribution of $V \sin i$ for the Pleiades stars with those obtained from the $q$ Maxwellian and the standard Maxwellian functions. Fig. 3 shows the best fits for the histogram of the observed distribution of normalized $V \sin i$ for the whole $B-V$ interval (case A in Table 1). The dashed line represents the distribution calculated from the $q$-Maxwellian function with $q=1.36$. The dotted line is the standard Maxwellian function. The number in parenthesis is the amount of stars in the sample. The distribution of observed $V \sin i$ is clearly more adequately fitted by the $q$-Maxwellian function. Such a behaviour is also observed for the other $B-V$ intervals represented in Table 1 , namely, cases $\mathrm{B}$ and $\mathrm{C}$.

Finally, in Fig. 4 we present the behaviour the parameter $\sigma$, the width of the $q$-Maxwellian, as a function of the parameter $q$ for two intervals of $B-V$ (case B). It is clear that, at least for the present stellar sample, the standard Maxwellian $(q=1)$ for stars with $B-V$ between 0.4 and 0.9 (left panel) is in the rejection region or outside the curve which delineates 0.05 significance level. For the second interval $0.9-1.40$ (right panel), the Maxwellian lies just in the frontier of the 0.05 significance level curve. This clearly shows that, at least at this significance level, the behaviour of the distribution of rotational velocity of stars of the Pleiades in these two $B-V$ interval is non-Maxwellian.

\section{Discussion and conclusions}

The most important result of the present work is that, based on the statistical analysis we carried out of the rotational velocities of stars in the open cluster Pleiades, the $q$-Maxwellian distribution function is a more feasible function to control the observed $V \sin i$ distributions. For the entire sample, the value of $q$ is found to be $1.36_{-0.05}^{+0.03}$, clearly different of $q=1$ of the Maxwellian distribution. This is not entirely surprising considering that the star formation and evolution process is not an isolated phenomenon, as underlined in the Introduction of the present study, this being particularly true for stars in clusters.

For instance, it is believed that the initial angular momentum is established during the early phase of accretion of gas to the new born star via locking of stellar rotational velocity to the angular velocity of the circumstellar accretion disk. For high accretion rate through the disk, the radius where the stellar magnetosphere links to the disk is smaller, its Keplerian rotation speed is high and, consequently, the 


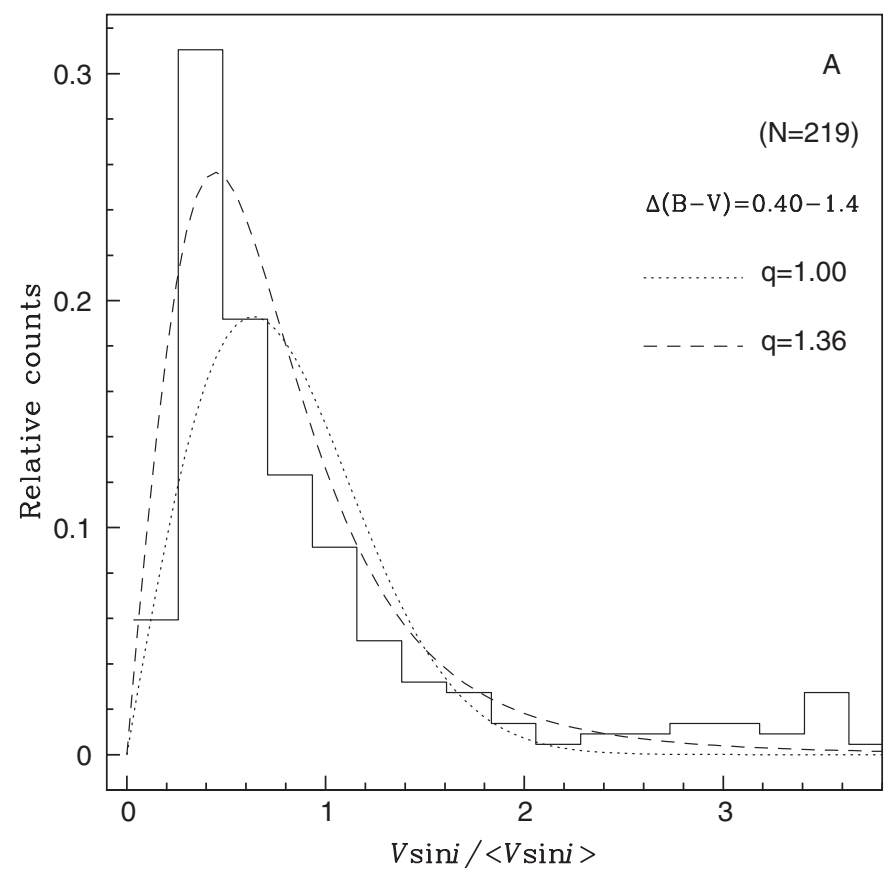

Fig. 3. The distribution of rotational velocities for the Pleiades stars. The histogram represents the observed distribution of normalized $V \sin i$. The dashed line represents the distribution calculated from the $q$-Maxwellian function. The dotted line is the standard Maxwellian function.
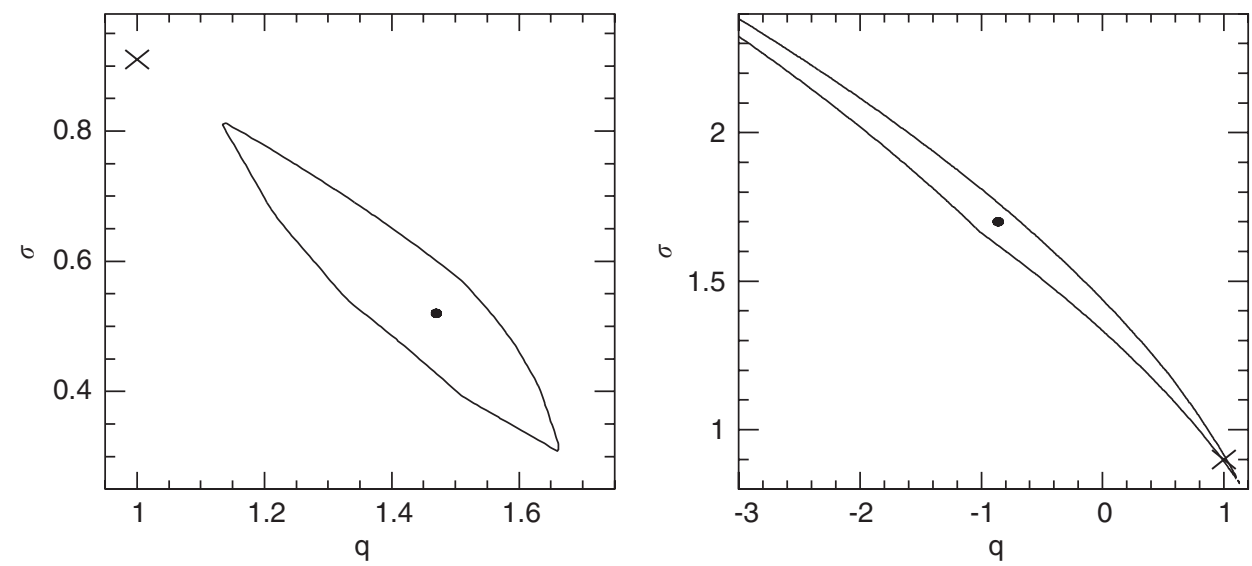

Fig. 4. The rejection region (outside the curve) of the null hypothesis that the $V \sin i$ distributions are drawn from the $q$-Maxwellian functions at 0.05 significance level for stars in case B with $B-V$ intervals $0.40-0.90$ (left panel) and $0.90-1.40$ (right panel). In both panels, the crosses $(\times)$ represent the best $\sigma$ for the standard Maxwellian function and the dots $(\bullet)$ represent the maximum probabilities for $q$ and $\sigma$ values.

rotational velocity of the star will also be higher. Therefore, in star formation regions where the density is high, so will be the accretion rate and one expects, on average, the rotational velocity to be higher as well. In support of this, the distribution of rotation velocities of B stars in the dense Orion Nebula cluster is observed to be shifted toward higher values when compared with stars found in much lower density regions of the Orion complex [29]. This process would explain the differences found, for example, in the rotational behaviour of cluster stars and field stars [30]. These arguments, as well as the observations, not only indicate that stars born in dense clusters rotate more rapidly, but that, the rotational velocity of a star is the result of a complex 
process. It involves, for instance, a strong interaction with the environment where the star was formed and where the star is now evolving, besides the angular momentum loss via stellar winds and angular momentum transfer in the stellar interior.

Another element to be considered as we look into the present result, in particular for $q \neq 1$, is the fact that we are analysing the projected rotational velocity. There is no insurance that the stars rotation axes are randomly orientated since the star final orientation of the rotation axis may depend on both the star formation process and its location in the Galaxy.

Finally, the fact that $q \neq 1$ for the whole sample may not come as total surprise if we consider that it has been shown both theoretical and observationally by several authors that in different astrophysical contexts involving the long-range nature of gravity [31,32], Tsallis non-extensive statistics gives excellent results.

A more unexpected result is the trend observed in which the $q$ value of the distribution decreases with increasing $B-V$ colour index. This behaviour of the $q$ value of the $V \sin i$ distribution seems to point for a clear dependence of rotation upon stellar mass. Let us recall that in stellar clusters like the Pleiades, stars present similar physical parameters except rotation and mass. In this sense, the behaviour of $q$ in the present analysis would seem to reflect the effect of stellar mass on rotation. Evidently, this deserves further investigation which we plan to carry out in future works by studying other stellar clusters and field stars with different physical parameters as metallicity, age and masses. We can also test this hypothesis by examining the distribution of rotational velocities for evolved stars, since we know that for these stars, other factors, besides the close relation between mass and rotation, are present. For instance, the rotational velocity of red giant stars depends, among others, on the depth of the convective zone, magnetic breaking, mass loss and tidal interactions. By analysing the behaviour of rotation along an evolutionary sequence from the star birth-line to the red giant stage, it will be possible to study the $q$ parameter as a function of time. Such an analyses may help us to understand where, in time, different physical processes are affecting stellar rotation and, by consequence, the evolution of the stellar angular momentum evolution.

The present study, which should be regarded as a preliminary attempt, seems to point to a fundamental aspect: for the study of the nature and the evolutionary behaviour of the distribution of stellar rotational velocity, it is necessary to take into account the different mechanisms that may play a role on rotation once stars are formed, which, very probably, affect rotation strength and axis orientation.

\section{Acknowledgements}

This work has been supported by continuous grants from the CNPq Brazilian Agency. We also acknowledge financial support of the Rio Grande do Norte FAPERN Agency. It is a pleasure to thank C. Tsallis for a careful reading of the manuscript and valuable suggestions for improvements in this work. We express our thanks to the referees for the careful reading and constructive comments on the original version of this paper. B. B. Soares thanks the CNPq for Grant 140458/00-0 and J. D. do Nascimento Jr. acknowledges the CNPq/PROFIX Grant 540461/01-6.

\section{References}

[1] O. Struve, Pop. Astr. 53 (1945) 202.

[2] S. Chandrasekhar, G. Münch, Astrophys. J. 111 (1950) 142.

[3] A.J. Deutsch, in: A. Slettebak (Ed.), Stellar Rotation, IAU Colloquium, Reidel, Dordrecht, 1970, p. 207.

[4] M.M. Dworetsky, Astrophys. J. 28 (1974) 101.

[5] S.C. Wolff, S. Edwards, G.W. Preston, Astrophys. J. 252 (1982) 322.

[6] A. Koenigl, Astrophys. J. 370 (1991) L39.

[7] F. Shu, J. Najita, E. Ostriker, F. Wilkin, S. Ruden, S. Lizano, Astrophys. J. 429 (1994) 781.

[8] J.R. De Medeiros, J.R.P. Da Silva, M.R.G. Maia, Astrophys. J. 578 (2002) 943.

[9] L. Siess, M. Livio, Mon. Not. R. Astron. Soc. 304 (1999) 925.

[10] J.R. De Medeiros, J.C. Carvalho, B. Soares, C. da Rocha, M.R.G. Maia, Astron. Astrophys. 358 (2000) 113.

[11] G. Burki, A. Maeder, Astron. Astrophys. 57 (1977) 401.

[12] R. Silva, A.R. Plastino, J.A.S. Lima, Phys. Lett. A 249 (1998) 401.

[13] C. Tsallis, J. Stat. Phys. 52 (1988) 479. 
[14] V.H. Hamity, D.E. Barraco, Phys. Rev. Lett. 76 (1996) 4664.

[15] A.K. Rajagopal, Phys. Rev. Lett. 76 (1996) 3469.

[16] A. Lavagno, G. Kaniadakis, M. Rego-Monteiro, P. Quarati, C. Tsallis, Astr. Lett. Comm. 34 (1998) 449.

[17] A. Taruya, M. Sakagami, Physica A 307 (2002) 185.

[18] D.H. Zanette, P.A. Alemany, Phys. Rev. Lett. 75 (1995) 366.

[19] G. Kaniadakis, A. Lavagno, P. Quarati, Phys. Lett. B 369 (1996) 308.

[20] J.A.S. Lima, R. Silva, J. Santos, Astron. Astrophys. 396 (2002) 309.

[21] C. Tsallis, S.V.F. Levy, A.M.C. Souza, R. Maynard, Phys. Rev. Lett. 75 (1995) 3589 Erratum: Phys. Rev. Lett. 77 (1996) 5442.

[22] C. Tsallis, Braz. J. Phys. 29 (1999) 1.

[23] S. Abe, A. Okamoto (Eds.), Nonextensive Statistical Mechanics and Its Applications, Springer, Heidelberg, 2001.

[24] J.A.S. Lima, A.R. Plastino, Braz. J. Phys. 30 (2000) 176.

[25] V. Latora, A. Rapisarda, C. Tsallis, Physica A 305 (2002) 129.

[26] A. Campa, A. Giansanti, D. Moroni, C. Tsallis, Phys. Lett. A 286 (2001) 251.

[27] R.P. Kraft, in: G.H. Herbig (Ed.), Spectroscopic Astrophysics. An Assessment of the Contributions of Otto Struve, University of California Press, Berkeley, 1970, p. 385.

[28] D. Queloz, S. Allain, J.-C. Mermilliod, J. Bouvier, M. Mayor, Astron. Astrophys. 335 (1998) 183.

[29] S.E. Strom, S.C. Wolff, D.H.A. Dror, Astrophys. J. 129 (2005) 809.

[30] C.H.F. Melo, L. Pasquini, J.R. De Medeiros, Astron. Astrophys. 375 (2001) 851.

[31] A.R. Plastino, A. Plastino, Phys. Lett. A 174 (1993) 384.

[32] A.R. Plastino, A. Plastino, Braz. J. Phys. 29 (1999) 79. 